\title{
Efficacy of high dose steroid therapy in children with severe acute transverse myelitis
}

Service de Neurologie, Département de Pédiatrie, Cliniques Universitaires

Saint-Luc, Université Catholique de

Louvain, Avenue

Hippocrate 10, 1200

Bruxelles, Belgium

P Defresne

H Kadhim

G Sébire

Unité de

Neuropathologie,

Service

d'Anatomopathologie, CHU

Brugmann-HUDERF, ULB, Bruxelles, Belgium

$\mathrm{H}$ Kadhim

Service de Neurologie, Département de Pédiatrie et INSERM U292, Service

d'Epidémiologie, Hôpital de Bicêtre,

Université Paris XI, Le Kremlin-Bicêtre,

France

L Meyer

M Tardieu

$P$ Landrieu

Service de Pédiatrie, Clinique de

l'Espérance,

Montegnée, Belgium

E Scalais

Service de Pédiatrie, Centre Hospitalier de Luxembourg, Luxembourg

C Nuttin

Service de Pédiatrie, Clinique de Jolimont, La Louvière, Belgium B De Bont

Pediatric Department, Galway University

Hospital, University of Galway, Galway,

Ireland

G Loftus

Correspondence to:

Dr P Defresne

pierre.defresne@freebel.net

Received 13 October 2000 and in revised form

13 February 2001

Accepted 11 April 2001

P Defresne, L Meyer, M Tardieu, E Scalais, C Nuttin, B De Bont, G Loftus, P Landrieu, H Kadhim, G Sébire

\begin{abstract}
No effective treatment has been demonstrated for patients with acute transverse myelopathy. In a multicentre controlled study, 12 children with severe acute transverse myelopathy were treated with intravenous methylprednisolone (IVMP) and compared with a historical group of 17 patients. The treatment had a significant effect on the proportion of patients walking independently at 1 month and on the proportion with full recovery at 1 year, with no differences in the frequency of complications between the two groups.

( $F$ Neurol Neurosurg Psychiatry 2001;71:272-274)
\end{abstract}

Keywords: steroids: acute transverse myelopathy: encephalomyelitis

Acute transverse myelopathy is a rare and severe disorder which usually involves the sensory and motor tracts of the spinal cord. The incidence has been estimated at 1.34 /million in the Israeli population ${ }^{1}$ but there has been no specific evaluation of the paediatric incidence. It is characterised by rapid onset of paraplegia or tetraplegia, loss of sensibility, and sphincter disturbances. The disease leads to permanent disability in about $40 \%$ to $60 \%$ of patients, based on findings from the main series. ${ }^{1-6}$ There is no evidence that any effective treatment exists. The presumed immune mediated pathophysiological mechanism for the disease, ${ }^{7}$ the high risk of residual neurological deficits, and reports of potential benefit of corticosteroids in acute disseminated encephalomyelitis, ${ }^{8}$ prompted us to evaluate the effects of high dose intravenous methylprednisolone (IVMP). In 1996, we performed an open pilot study applying this treatment to five patients. The outcome of these patients was compared with that of a historical group of 10 patients who did not receive IVMP treatment. ${ }^{9}$ The results suggested that IVMP could shorten the duration of the disease and improve the outcome at 1 year. In the light of these promising results, we decided to expand the series using the same methods. We chose to treat patients displaying complete flaccid paralysis of the legs, which is associated with a high risk of poor outcome. ${ }^{5} 10$
Patients and methods

Twenty nine children, including the 15 patients from the original series, ${ }^{9}$ were admitted between 1975 and 1999 to several European centres with the diagnosis of severe acute transverse myelitis and presenting with at least a complete flaccid paralysis of the lower limbs. All the children (mean age 8.6 years; range 1 to 14 years) fulfilled the classic criteria for the diagnosis of acute transverse myelopathy ${ }^{14611}$ : briefly, no antecedent neurological illness, acute onset of bilateral spinal cord dysfunction, and exclusion of patients with spinal trauma or irradiation. Compressive spinal cord lesion was also excluded by MRI (22 patients), myelography (two patients), and Queckenstedt test (two patients). These investigations were not performed in the remaining three patients presenting with myelopathy associated with optic neuritis. Patients in whom neurological symptoms had recurred were not enrolled (except one patient who had a single relapse followed by full recovery and no recurrence during a 3 year follow up period). Twelve were treated with IVMP at a dosage of $1 \mathrm{~g} / 1.73 \mathrm{~m}^{2} /$ day for three (five patients) or five consecutive days (seven patients), followed in 10 of the 12 patients by oral prednisone $(1 \mathrm{mg} / \mathrm{kg} /$ day $)$ for a total period of 2 (eight patients) or 3 weeks (two patients). One patient received an initial dose of 0.5 $\mathrm{g} / 1.73 \mathrm{~m}^{2}$. Only children whose parents gave informed consent took part in the study. Their outcome was compared with that of a historical group (17 patients), receiving either no specific treatment or low dose corticosteroids which, according to previous studies, do not affect the outcome. ${ }^{4}{ }^{6}$ For the group as a whole, the mean follow up duration was 3.9 years (range 1 to 15 years). The main outcome criteria were: (1) the proportion of patients with full recovery, with motor recovery, and with sphincter recovery within 12 months; (2) the proportion of patients able to walk independently after 1 month; (3) the proportion of patients with complications - namely, corticosteroid adverse effects (persistent hyperglycaemia, high blood pressure), bedsores, and urinary tract infections. We also studied the median time to independent walking and the quality of recovery. The quality of recovery after 1 year was evaluated using Paine's scale": (1) "normal": full recovery; (2) "good": gait essentially normal, 
Table 1 Characteristics of the two groups of patients

\begin{tabular}{|c|c|c|c|}
\hline & $\begin{array}{l}\text { IVMP group } \\
(n=12)\end{array}$ & $\begin{array}{l}\text { Control group } \\
(n=17)\end{array}$ & $p$ Value \\
\hline Year of observation (range) & 1994-9 & $1975-1998$ & \\
\hline Mean age (y (range)) & $9.5(2-14)$ & $8.0(1-14)$ & 0.4 \\
\hline Sex ratio $(M / F)$ & $8 / 4$ & $5 / 12$ & 0.05 \\
\hline \multicolumn{4}{|l|}{ Clinical features: } \\
\hline Back pain & $4 / 12$ & $7 / 17$ & 0.7 \\
\hline Hyperacute onset $(<12 \mathrm{~h})$ & $3 / 12$ & $4 / 17$ & $>0.999$ \\
\hline Supramedullary symptoms & $4 / 12$ & $5 / 17$ & $>0.999$ \\
\hline Complete flaccid paraplegia & $12 / 12$ & $17 / 17$ & 1.0 \\
\hline Sphincter disturbances & $11 / 12$ & $17 / 17$ & 0.4 \\
\hline Sensory level & $10 / 12$ & $15 / 17$ & $>0.999$ \\
\hline Increased CSF cell count & $8 / 12$ & $8 / 17$ & 0.45 \\
\hline Mean follow up (months) & 14.6 & 66 & 0.001 \\
\hline
\end{tabular}

IVMP=Intravenous methylprednisolone.

but mild urinary symptoms and/or minimal sensory and upper motor neuron signs; (3) "fair": mild spasticity but independent ambulation, urgency of micturition and/or constipation with some sensory signs; (4) "poor": unable to walk or severe gait disturbances, absence of sphincter control and sensory deficit. Percentages were compared using a $\chi^{2}$ or exact Fisher's test. The median time to independent walking was compared using the Kruskall-Wallis test. Student's $t$ test was used to compare the mean age and duration of follow up between the two groups.

\section{Results}

The clinical features of the patients are summarised in table 1 . The characteristics of both groups, especially those known to be of prognostic value, were identical - namely, back pain, ${ }^{4}$ hyperacute onset, ${ }^{46}$ and severity of initial course. $^{59}$ The mean time between disease onset and IVMP administration was 8.1 days (range 1 to 21 days). The proportion of patients who walked independently after 1 month was $66 \%$ (eight of 12) in the IVMP group versus $17.6 \%$ (three of 17 ) in the control group $(p=0.02)$. Full recovery within 1 year was seen in $54.6 \%$ of patients (six of 11 ) in the treated group versus $11.7 \%$ (two of 17 ) in the control group $(p=0.03)$. At 1 year, seven of 11 treated patients had achieved a complete motor recovery versus four of 17 in the control group $(p=0.05)$, and nine of 12 patients of the IVMP group had normal sphincter function versus three of 17 in the control group $(p=0.006)$. Among patients who recovered walking, the median time to independent walking was shorter in treated patients than in the control group (25 days $v 120$ days; $\mathrm{p}=0.05$ ). After 1 year, $75 \%$ of treated patients had a "normal" or "good" outcome versus $23.5 \%$ in the control group $(p=0.006)$. The proportion of patients with complications was identical in both groups (two of $12 v$ five of $17 ; \mathrm{p}>0.66$ ).

\section{Discussion}

These results demonstrate a favourable effect of IVMP on the outcome of severe acute transverse myelopathy in children. This treatment increased the proportion of patients with full recovery, accelerated the recovery of walking, and reduced the severity of sequelae.

These findings are in agreement with the high rate of recovery seen in an uncontrolled study using the same IVMP regimen in children with acute transverse myelopathy. ${ }^{12}$ Recent studies suggested that high dose steroid treatment might delay the onset of neurological progression for up to 6 months in some isolated demyelinating syndromes but that its protective effect is ultimately lost over several years of follow up. ${ }^{13}$ However, the long term effect of this treatment in acute transverse myelopathy, as well as in related conditions has yet to be ascertained. By contrast, two other studies showed that low dose corticosteroid treatment comparable with that used in our control group did not affect the outcome. ${ }^{4} 6$

The frequency of a good outcome after 1 year, in patients not treated with IVMP as reported in the literature, is about $40 \%$ to $60 \%$, irrespective of severity of disease. ${ }^{1-6}$ Our control group had a poorer outcome. This is due to the fact that only severely affected patients-namely, those presenting complete paraplegia (predictor of poor prognosis) - were included in this study because of the potential risks of IVMP. ${ }^{510}$ It is noteworthy that the recovery rate we found in our control group was similar to that reported in other series when using the same selection criteria. ${ }^{5}$

There had been some differences in the means of investigations between the two study groups. However, the earlier patients were as comprehensively investigated as the current patients. The two groups share the same classic diagnostic criteria for acute transverse myelopathy. On the other hand, those earlier patients had a fairly long follow up (mean 66 months). This makes the risk of relapsing or chronic progressive disease unlikely.

Acute transverse myelitis represents a heterogeneous group of conditions in which the postinfectious aetiology largely predominates, especially in children. ${ }^{2}$ It is most often considered as a localised form of acute demyelinating encephalomyelitis. ${ }^{10}$ The occurrence of occasional and relatively mild supramedullary symptoms in a few of our patients might therefore reflect some rostral extension of this continuum. On the other hand, the proportion of patients with supramedullary symptoms was similar in the two groups. This rules out the risk of unbalanced distribution. The control group showed a female predominance. In adults, women with acute transverse myelopathy have a tendency to show a high correlation with a future neurological progression to multiple sclerosis and might be regarded as carrying a worse prognosis. By contrast, the sex in childhood acute transverse myelopathy was not shown to influence the evolution of the disease. Moreover, the diagnosis of multiple sclerosis seems very unlikely in our young patients as: (1) multiple sclerosis is rare in childhood (2.7\% to $4.4 \%$ of cases of multiple sclerosis begin before the age of 16 years and only $3 \%$ of these children present initially with transverse myelitis) ${ }^{1415}$; (2) no recurrence was noted in our patients despite a long follow up period (except for one patient with a single relapse). In the only relevant study assessing the risk of evolution towards multiple sclerosis in childhood acute transverse myelopathy, no patient 
developed definite evidence of multiple sclero$\operatorname{sis}^{6}$; (3) CSF protein electrophoresis performed in our patients did not show any oligoclonal antibody synthesis. Oligoclonal bands in CSF were only studied in a few cases of childhood acute transverse myelopathy. ${ }^{2}$ In these cases, synthesis of oligoclonal immunoglobulins was detected very rarely. The sum of these findings provides substantial arguments for distinct repartition of aetiopathogenic mechanisms in children compared with adults with acute transverse myelopathy. The disease results from different pathophysiological mechanisms in which the postinfectious aetiology largely predominates in children, whereas in adults, other conditions, such as relapsing autoimmune diseases and spinal infarcts, prevail. ${ }^{2}$ Spinal cord infarction is unlikely in our patients as it is very rare in childhood. Moreover, our patients did not present any of the major predisposing factors such as cardiovascular diseases or coagulopathies. ${ }^{16}$

The aetiology of ATM in childhood remains obscure, but a postinfectious autoimmune process is suggested by the seasonal clustering, association with a previous infection, the frequent inflammatory reactions detected in the CSF, and by anatomical studies showing infiltration of the spinal white matter by immune cells. ${ }^{7}$ The myelin sheath seems to be the target of the pathological process, as suspected clinically by the association with supramedullary demyelinating symptoms, such as optic neuritis, and confirmed by anatomical studies showing areas of spinal cord demyelination. Moreover, peripheral blood lymphocytes from patients with acute transverse myelopathy show a significant proliferative response when cultured in vitro in the presence of myelin proteins such as myelin basic protein. ${ }^{7}$ Thus, acute transverse myelopathy might be the consequence of a cell mediated autoimmune response directed against a component of the myelin sheath. The efficacy of IVMP in related diseases such as optic neuritis ${ }^{17}$ and multiple sclerosis $^{18}$ led us to study its use in the treatment of severe paediatric forms of acute transverse myelopathy. The mechanism of action of IVMP has not been fully elucidated. In peripheral blood, IVMP induces a transient lymphopenia with a significant reduction in CD4 positive cells. ${ }^{19} 20$ An anti-inflammatory effect has also been demonstrated in vitro in
CNS cells. For example, glucocorticoids down regulate the production of cytokines such as interleukin- 6 and TNF- $\alpha$ by microglial cells and peripheral macrophages. ${ }^{21}$

In our opinion and taking into account the results of our study, the likelihood of a beneficial effect of IVMP in childhood acute transverse myelopathy is so strong that a double blind placebo controlled study would raise some concerns.

1 Berman M, Feldman S, Alter M, et al. Acute transverse myelitis: incidence and etiologic considerations. Neurology 1981;31:966-71.

2 Knebusch M, Strassburg HM, Reiners K. Acute transverse myelitis in childhood: nine cases and review of the literature. Dev Med Child Neurol 1998;40:631-9.

3 Paine RS, Byers RK. Transverse myelopathy in childhood. Am ₹ Dis Child 1953;85:151-63.

4 Ropper AH, Poskanzer DC. The prognosis of acute and Ropper AH, Poskanzer DC. The prognosis of acute and
subacute transverse myelopathy based on early signs and subacute transverse myelopathy base
symptoms. Ann Neurol 1978;4:51-9.

5 Kymptoms. Ann Neurol 1978;4:51-9. acute transverse myelitis. Acta Neurol Scand 1998;98:60-3.

6 Dunne K, Hopkins IJ, Shield LK. Acute transverse myelopathy in childhood. Dev Med Child Neurol 1986;28: 198-204.

7 Abramsky O, Teitelbaum D. The autoimmune features of acute transverse myelopathy. Ann Neurol 1977;2:36-40.

8 Pasternak JF, De Vivo DC, Prensky AL. Steroid-responsive encephalomyelitis in childhood. Neurology 1980;30:481-6.

9 Sébire G, Hollenberg H, Meyer L, et al. High dose methylprednisolone in severe acute transverse myelopathy. Arch Dis Child 1997;76:167-8.

10 Al Deeb SM, Yaqub BA, Bruyn GW, et al. Acute transverse myelitis: a localized form of postinfectious encephalomyelitis. Brain 1997;120:1115-22.

11 Jeffery DR, Mandler RN, Davis LE. Transverse myelitis: retrospective analysis of 33 cases, with differentiation of cases associated with multiple sclerosis and parainfectious events. Arch Neurol 1993;50:532-5.

12 Lahat E, Pillar G, Ravid S, et al. Rapid recovery from transverse myelopathy in children treated with methylprednisolone. Pediatr Neurol 1998;19:279-82.

13 Kinkel RP. Methylprednisolone. In: Rudich RA, Goodkin DE, eds. Multiple sclerosis therapeutic. 1st ed. London: Martin Duvitz, 1999:357-8.

14 Duquette P, Murray TJ, Pleines J, et al. Multiple sclerosis in childhood: clinical profile in 125 patients. $\mathcal{F}$ Pediatr 1987;111:359-63.

15 Ghezzi A, Deplano V, Faroni J, et al. Multiple sclerosis in childhood: clinical features of 149 cases. Mult Scler 1997;3: 43-6.

16 Cheshire WP, Santos CC, Massey EW, et al. Spinal cord infarction: etiology and outcome. Neurology 1996;47:32130

17 Beck RW, Clearly PA, Anderson MM, et al. A randomised, controlled trial of corticosteroids in the treatment of acute optic neuritis. N Engl f Med 1992;326:581-8.

18 Goodin DS. The use of immunosuppressive agents in the reatment of multiple sclerosis: a critical review. Neurology 1991;41:980-5.

19 Miller JJ. Prolonged use of large intravenous pulses in the rheumatic diseases of children. Pediatrics 1980;65:989-94.

20 Silverman ED, Myones BL, Miller JJ. Lymphocytes subpopulation alterations induced by intravenous megasubpopulation alterations induced by intravenous mega-
dose pulse methyl-prednisolone. $\mathcal{F}$ Rheumatol 1984;11:28790 .

21 Sebire G, Delfraissy JF, Demotes-Mainard J, et al. nterleukin-13 and interleukin-4 act as interleukin-6 inducers in human microglial cells. Cytokine 1996;8:63641 